\title{
Effect of the lattice alignment on Bloch oscillations of a Bose-Einstein condensate in a square optical lattice
}

\author{
M.-C. Chung ${ }^{1, a}$ and A.R. Kolovsky ${ }^{2,3}$ \\ 1 Max-Planck-Institut für Physik komplexer Systeme, 01187 Dresden, Germany \\ 2 Kirensky Institute of Physics, 660036 Krasnoyarsk, Russia \\ 3 Siberian Federal University, 660041 Krasnoyarsk, Russia
}

Received 5 November 2007 / Received in final form 26 February 2008

Published online 4 April 2008 - C) EDP Sciences, Società Italiana di Fisica, Springer-Verlag 2008

\begin{abstract}
We consider a Bose-Einstein condensate of ultracold atoms loaded into a square optical lattice and subject to a static force. For vanishing atom-atom interactions the atoms perform periodic Bloch oscillations for arbitrary direction of the force. We study stability of these oscillations for non-vanishing interactions, which is shown to depend on an alignment of the force vector with respect to the lattice crystallographic axes. If the force is aligned along any of the axes, the mean field approach can be used to identify the stability conditions. On the contrary, for a misaligned force one has to employ the microscopic approach, which predicts periodic modulation of Bloch oscillations in the limit of a large forcing.
\end{abstract}

PACS. 03.75.Lm Bose-Einstein condensates in periodic potentials - 03.75.Kk Dynamic properties of condensates

\section{Introduction}

Due to a virtually perfect experimental control over parameters, ultracold neutral atoms in optical lattices have been intensively used for studying a variety of phenomena of the condensed matter physics, including the transport phenomena like Landau-Zener tunnelling $[1,2]$, WannierStark ladder [3], and Bloch oscillations [3-6]. A number of exciting experimental results were reported during the last decade, which also stimulated the progress in the theory [7-10]. As concerns applications, one should distinguish the latter problem of Bloch oscillations (BO) as particularly important, because $\mathrm{BO}$ provide a tool for a precision measurement of the gravitational field and interatomic interaction constant $[5,6]$.

Until quite recently almost all theoretical, numerical and experimental studies of BO concerned $1 \mathrm{D}$ or quasi 1D lattices (see Refs. [8-10] for the contemporary reviews). Nowadays one observes a growing interest in $\mathrm{BO}$ in multidimensional lattices [10-15]. In the single-particle approach this problem was considered in references $[11,12]$. It was shown that an increase of the lattice dimensionality introduces new effects not present in the 1D lattice. Some predictions of these works were later on confirmed in the experiment with the array of optical guides [14], where one uses a formal analogy between the Maxwell and Schrödinger equations. The experiments with a BoseEinstein condensate (BEC) of interacting atoms addresses the further questions [15], in particular, the question about the stability of multidimensional BO. Indeed, it is known

\footnotetext{
a e-mail: mccchung@pks.mpg.de
}

that a BEC in optical lattices can be dynamically unstable, which quantum-mechanically means decoherence of the BEC [16]. In the present work we study the conditions under which the dynamical instability is suppressed and, hence, multidimensional $\mathrm{BO}$ are stable. Unlike 1D lattices, these conditions are shown to involve an alignment of the static force vector with respect to the crystallographic axes of the lattice. We also argue in the work that by changing the angle between the primary lattice vectors and the force vector one may observe a transition from the mean-field to the microscopic Bloch dynamics.

\section{The Hamiltonian}

To simplify the equations we shall consider the twodimensional case throughout the paper - generalization of the results in three dimensions is straightforward. The Bose-Hubbard Hamiltonian of atoms in the tilted 2D lattice reads,

$$
\begin{aligned}
\widehat{H}= & -\frac{J_{x}}{2} \sum_{m, l}\left(\hat{a}_{m+1, l}^{\dagger} \hat{a}_{m, l}+\text { h.c. }\right) \\
& -\frac{J_{y}}{2} \sum_{m, l}\left(\hat{a}_{m, l+1}^{\dagger} \hat{a}_{m, l}+\text { h.c. }\right) \\
& +\frac{W}{2} \sum_{m, l} \hat{n}_{m, l}\left(\hat{n}_{m, l}-1\right) \\
& +d \sum_{m, l}\left(F_{x} m+F_{y} l\right) \hat{n}_{m, l},
\end{aligned}
$$


where $J_{x, y}$ are the hopping matrix elements in $x$ and $y$-directions, $W$ microscopic atom-atom interaction constant, $d$ lattice period, and $F_{x, y}$ the projections of the static force vector on the lattice axes. The Hilbert space of (1) is spanned by the Fock states $|\mathbf{n}\rangle \equiv\left|n_{m, l}\right\rangle$, where $\sum_{m, l} n_{m, l}=N$ - the total number of atoms. (Since in the coordinate representation the Fock states are given by the symmetrized product of the localized Wannier functions, we shall refer to this basis as the Wannier basis.) The translational invariance of the system, broken by the static term, can be actually recovered by using the gauge transformation [7]. Then the Hamiltonian (1) takes the form

$$
\begin{aligned}
\widehat{H}(t)= & -\frac{J_{x}}{2} \sum_{m, l}\left(e^{-i \omega_{x} t} \hat{a}_{m+1, l}^{\dagger} \hat{a}_{m, l}+h . c .\right) \\
& -\frac{J_{y}}{2} \sum_{m, l}\left(e^{-i \omega_{y} t} \hat{a}_{m, l+1}^{\dagger} \hat{a}_{m, l}+h . c .\right) \\
& +\frac{W}{2} \sum_{m, l} \hat{n}_{m, l}\left(\hat{n}_{m, l}-1\right)
\end{aligned}
$$

where $\omega_{x, y}=d F_{x, y} / \hbar$ are the Bloch frequencies associated with $x$ and $y$ component of the static force. We also note that in stead of the Wannier basis one can use the quasimomentum Fock basis $|\mathbf{q}\rangle \equiv\left|q_{p, k}\right\rangle$ for the Hamiltonian (2), which we shall refer to as the Bloch basis. (Needless to say that in the coordinate representation the quasimomentum Fock states are given by the symmetrized product of the extended Bloch functions.) Formally this corresponds to the canonical transformation

$$
\hat{b}_{p, k}=\frac{1}{L} \sum_{m, l} \exp \left[-i \frac{2 \pi}{L}(m p+k l)\right] \hat{a}_{m, l},
$$

which implicitly assumes the periodic boundary conditions.

Through the paper we shall illustrate the analytical results by simulating $\mathrm{BO}$ in a finite $2 \mathrm{D}$ lattice and it is an appropriate place here to comment on the choice of the system parameters. This choice is determined by two criteria: we want our finite system to reproduce the main features of an infinite system but, to minimize computational efforts, to be as small as possible. It is an advantage of the periodic boundary conditions that already the $3 \times 3$ lattice can satisfy the former condition. For example, if equation (8), which we shall discuss later on in Section 3.2 and which refers to an infinite lattice, is applied to the lattice with $L=3$ sites, it will give an error of less than 5 percents. As concerns the filling factor $\bar{n}=N / L^{2}$, we keep it close to unity, which corresponds to its typical value in the contemporary laboratory experiments. Note that for the considered in the work ratio $W / J_{x, y}<1$ the exact value of the filling factor does not matter. It becomes important only if one considers larger interactions, where the system may show different types of the superfluid to Mott-insulator quantum phase transitions. (For example, for integer $N / L$ and not equal hopping matrix elements the system can be super-fluid in one direction but insulating in the orthogonal direction.) Since our subject is $\mathrm{BO}$, we intentionally stay far from insulating phase by choosing $W$ smaller than either of the hopping matrix elements.

A remark about the magnitude of the static force is in turn. Formally, i.e., within the frame of the Bose-Hubbard model, the static force can be arbitrary large. However, when referring to the original system of cold atoms in an optical lattice, the force magnitude is restricted from above by requiring negligible Landau-Zener transitions. The latter condition is satisfied by assuming $\Delta>d F$, where $\Delta$ is the energy gap separating the ground Bloch band from the rest of the spectrum.

To conclude this section we mention that although a small system with $L=3$ or $L=4$ sites along one axis, used in the numerical simulations below, is capable to reproduce the features of infinite $2 \mathrm{D}$ lattice, we cannot completely avoid the presence of finite-size effects. In what follows we shall explicitly indicate which of the numerical results have general validity and which are artifacts due to the system finite size.

\section{Bloch dynamics}

\subsection{Misaligned force}

We begin with the case of a strong misaligned force $d F_{x}, d F_{y} \gg J_{x, y}>W[17]$. In order to illuminate situation, we simulate BO by numerically solving the timedependent Schrödinger equation with the Hamiltonian (2) for the specified initial conditions. As those we consider the superfluid state with $q_{p, k}=N \delta_{p, 0} \delta_{k, 0}$, which approximates the ground state of the system for $\mathbf{F}=0$ and $W<J_{x, y}$. (Substitution of this state by the exact ground state practically does not affect the final result.) Figure 1 shows the numerical results for the $3 \times 3$ lattice with 7 atoms inside. The lower panel in Figure 1 depicts the kinetic energy of the atoms, the upper and middle panels show the order parameters $e_{x}(t)$ and $e_{y}(t)$ defined as [18]

$$
e_{x}(t)=-\frac{1}{N} \operatorname{Re}\left[\left\langle\Psi(t)\left|\sum_{m, l} \hat{a}_{m+1, l}^{\dagger} \hat{a}_{m, l}\right| \Psi(t)\right\rangle\right] .
$$

(By replacing the operators in the bracket in equation (3) with $\sum \hat{a}_{m, l+1}^{\dagger} \hat{a}_{m, l}$ one obtains a similar expression for the order parameter $e_{y}(t)$.) It is seen in the figure that BO persist in time but are modulated with some characteristic period. We have checked that this period is not affected by increasing the lattice size and, hence, the displayed result has a general validity.

To prove that $\mathrm{BO}$ in the misaligned lattice are stable in the limit of strong forcing and to identify the modulation period we proceed as follows. First we introduce the new wave function $|\widetilde{\Psi}(t)\rangle$ through the relation $|\Psi(t)\rangle=\widehat{U}_{0}(t)|\widetilde{\Psi}(t)\rangle$, where $\widehat{U}_{0}(t)$ is the evolution operator for vanishing atom-atom interactions. The function 

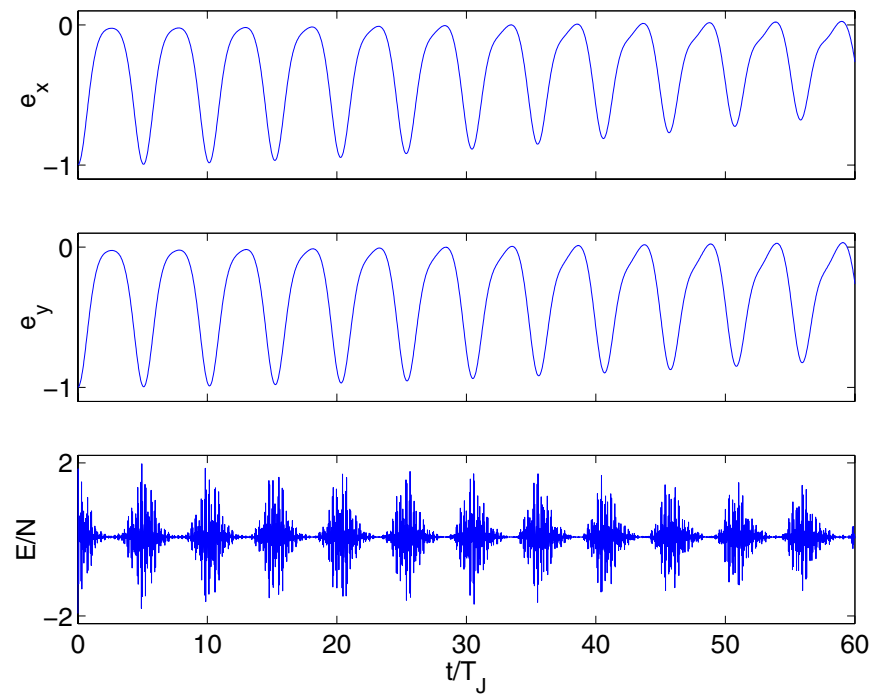

Fig. 1. Bloch oscillations of condensed atoms in the 2D lattice: the order parameter $e_{x}(t)$ (top), $e_{y}(t)$ (middle), and the mean kinetic energy (bottom). The system parameters are $N=7, L=3$ (periodic boundary conditions), $J_{x}=J_{y}=J$, $W=0.2 J, F d=20 J$, and $\mathbf{F} / F=(\sqrt{2 / 5}, \sqrt{3 / 5})$. The time is measured in units of the tunnelling period $T_{J}=2 \pi \hbar / J$.

$|\widetilde{\Psi}(t)\rangle$ obviously obeys the equation,

$i \hbar \frac{\partial|\widetilde{\Psi}(t)\rangle}{\partial t}=\frac{W}{2} \widehat{U}_{0}^{\dagger}(t)\left(\sum_{m, l} \hat{n}_{m, l}\left(\hat{n}_{m, l}-1\right)\right) \widehat{U}_{0}(t)|\widetilde{\Psi}(t)\rangle$.

On the other hand, the explicit form of the evolution operator is given by $\widehat{U}_{0}(t)=\widehat{T}^{\dagger} \widehat{D}(t) \widehat{T}$, where the unitary operator $\widehat{T}$ represents the transformation from the Wannier basis $|\mathbf{n}\rangle$ to the Bloch basis $|\mathbf{q}\rangle$ and the matrix of the operator $\widehat{D}(t)$ is diagonal in the Bloch basis,

$$
\begin{aligned}
\langle\mathbf{q}|D(t)| \mathbf{q}\rangle= & \exp \left[i \frac{J_{x}}{d F_{x}} \sum_{i=1}^{N} \sin \left(\frac{2 \pi p_{i}}{L}-\omega_{x} t\right)\right. \\
& \left.+i \frac{J_{y}}{d F_{y}} \sum_{i=1}^{N} \sin \left(\frac{2 \pi k_{i}}{L}-\omega_{y} t\right)\right] .
\end{aligned}
$$

Note that the operator (5) tends to the identity operator for $F_{x}, F_{y} \rightarrow \infty$. Substituting $\widehat{U}_{0}(t)$ in equation (4) by identity matrix and noting that the interaction energy operator is diagonal in the Wannier basis with integer entries, $\left\langle\mathbf{n}\left|\sum_{m, l} \hat{n}_{m, l}\left(\hat{n}_{m, l}-1\right)\right| \mathbf{n}\right\rangle=\sum_{m, l} n_{m, l}^{2}-N$, we conclude that the time evolution of the wave function $|\widetilde{\Psi}(t)\rangle$ is periodic with the period $T_{W}=2 \pi \hbar / W$ [19]. Coming back to the original wave function this result means the periodic modulation of $\mathrm{BO}$ with the frequency $\omega_{W}=W / \hbar$. It is worth stressing that the above proof assumes both $F_{x}$ and $F_{y}$ to be large and, hence, the case of aligned lattices is excluded.

\subsection{Aligned force}

Next we consider the situation where the force is aligned along one of the crystallographic axes (to be certain, the $y$-axis in what follows). Within the single-particle approach the static force $F_{y}$ would localize the atoms in the $y$-direction. Thus one may expect that if $F_{y}$ is large enough the atoms form separate BECs in the planes perpendicular to the force vector, weakly coupled together as a one-dimensional BEC chain. Introducing new operators $\hat{A}_{l}=\frac{1}{\sqrt{L}} \sum_{m} \hat{a}_{m, l}$ and $\hat{A}_{l}^{\dagger}$, the effective Hamiltonian reads

$$
\begin{aligned}
\widehat{H}_{\text {eff }}= & -J_{x} \sum_{l} \hat{A}_{l}^{\dagger} \hat{A}_{l}-\frac{J_{y}}{2} \sum_{l}\left(e^{-i \omega_{y} t} \hat{A}_{l+1}^{\dagger} \hat{A}_{l}+\text { h.c. }\right) \\
& +\frac{W_{\text {eff }}}{2} \sum_{l} \hat{N}_{l}\left(\hat{N}_{l}-1\right)
\end{aligned}
$$

where $W_{\text {eff }}=W / L$. Thus we have reduced the $2 \mathrm{D}$ problem to a $1 \mathrm{D}$ problem with the renormalized interaction constant. (If one considers 3D lattices, the renormalization is $W_{\text {eff }}=W / L^{2}$.) Moreover, since the mean number of atoms $\widetilde{N}$ in any site of the effective 1D system is given by $\bar{n} L$, the occupation numbers will be macroscopically large in the thermodynamic limit $N, L \rightarrow \infty, \bar{n}=N / L^{2}=$ const., which justifies the mean field approach.

The mean-field Hamiltonian of the system (6) reads (up to the irrelevant constant terms proportional to $N$ )

$$
H_{e f f}=-\frac{J_{y}}{2} \sum_{l}\left(e^{-i \omega_{y} t} A_{l+1}^{*} A_{l}+\text { h.c. }\right)+\frac{g}{2} \sum_{l}\left|A_{l}\right|^{4},
$$

where $A_{l}$ and $A_{l}^{*}$ are pairs of the canonically conjugated variables and the macroscopic interaction constant $g=W_{\text {eff }} \widetilde{N}=W \bar{n}$. Within the mean-field approach the border between stable and unstable (decaying) $\mathrm{BO}$ is know exactly $[16,20]$. Namely, for $J / F d \gtrsim 0.5$ the critical value of nonlinearity is a linear function of the static force magnitude, while for $J / F d \lesssim 0.5$ it additionally depends on the value of the hopping matrix elements:

$$
g_{c r} \approx\left\{\begin{array}{l}
0.33 F d, \quad F d \lesssim 2 J \\
0.1(F d)^{2} / J, F d \gtrsim 2 J .
\end{array}\right.
$$

Obviously, for a fixed nonlinearity $g$ the condition (8) can also be formulated as a condition on the critical magnitude $F_{c r}$ of the static force.

The microscopic analysis of $\mathrm{BO}$ in the aligned lattice confirms our working hypothesis. Choosing the parameters in such a way that the $1 \mathrm{D}$ mean-field $\mathrm{BO}$ are stable, we simulate $\mathrm{BO}$ of $N=7$ atoms in the $2 \mathrm{D}$ lattice with $L=3$. The dashed line in the upper panel of Figure 2 shows the dynamics of the order parameter $e_{x}(t)$. It is seen that $e_{x}(t) \approx-1,-$ therefore we indeed have in-plane BECs. We also note that the decay and revival of the order parameter $e_{y}(t)$ in the lower panel is an artifact due to the finite size of our lattice. We have checked numerically that increasing the lattice size modifies the revivals 

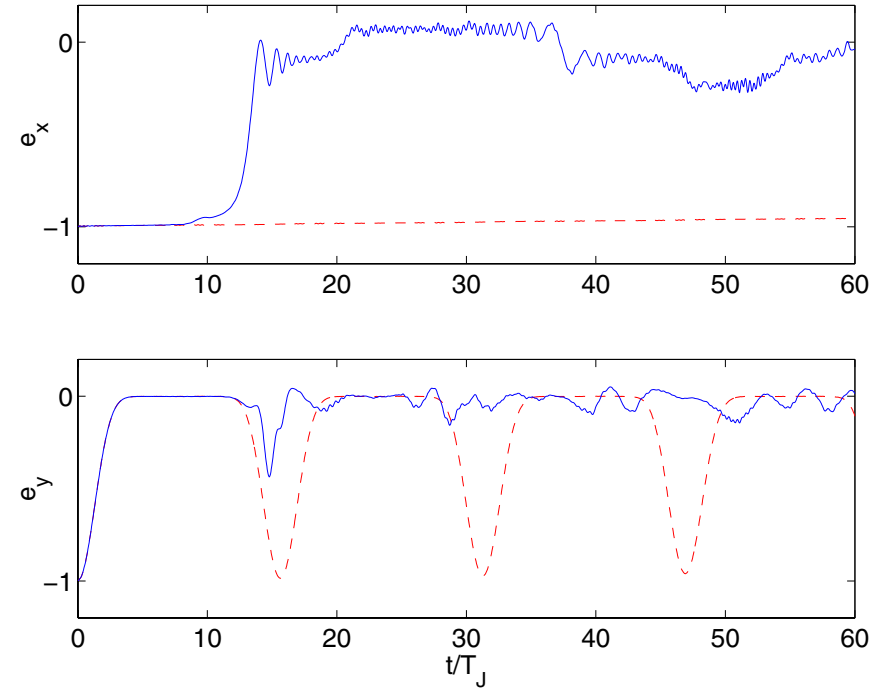

Fig. 2. (Color online) Dynamics of the order parameters for $\mathbf{F} / F=(0,1)$, dashed lines, and $\mathbf{F} / F=(0.001, \sqrt{1-0.001})$, solid lines. The other parameters are the same as in Figure 1.

according to the following equation,

$$
e_{y}(t)=-\exp \left(-2 \widetilde{N}\left[1-\cos \left(\frac{W_{\text {eff }} t}{\hbar}\right)\right]\right)
$$

which can be obtained analytically on the basis of the effective Hamiltonian (6) [10]. Because $W_{\text {eff }}=W / L$ and $\widetilde{N}=\bar{n} L$, one has $e_{y}(t)=-1$ in the thermodynamic limit.

The above analysis of $\mathrm{BO}$ in the aligned lattice relies on the reduction of a two-dimensional system to an effective one-dimensional mean-field problem. It should be especially stressed that this reduction is possible only if the dynamics of the reduced system is stable. If we choose the parameters in the unstable regime the situation becomes totally different. Figure 3 shows the numerical results for $F=0.2 \mathrm{~J} / d<F_{c r}$, where one-dimensional $\mathrm{BO}$ suffer from dynamical instability. Unlike in the stable regime, BO along $y$ direction now excite the transverse degree of freedom and we observe decay of the both order parameters towards zero. Thus no reduction to one dimension is possible.

\subsection{Slightly misaligned force}

Finally we briefly analyze an experimentally important situation of a small mismatch between the lattice axis and the static field vector, i.e., $F_{x} \ll F_{y}$. The solid lines in Figure 2 show the order parameters for the same $d F=20 J$ but $F_{x}=0.001 F$. Compared to the case $F_{x}=0$ (dashed lines in Fig. 2), we observe the destruction of BEC after time $t^{*} \approx 12 T_{J}$. This critical time can be understood in terms of the mean-field approach as well. Indeed, it is known that a stationary BEC is unstable for the quasimomentum $\kappa$ outside the first quarter of the Brillouin zone. Since the static force causes the linear growth of the quasimomentum, $\kappa_{x, y}(t)=\kappa_{x, y}+F_{x, y} t / \hbar$, the system always
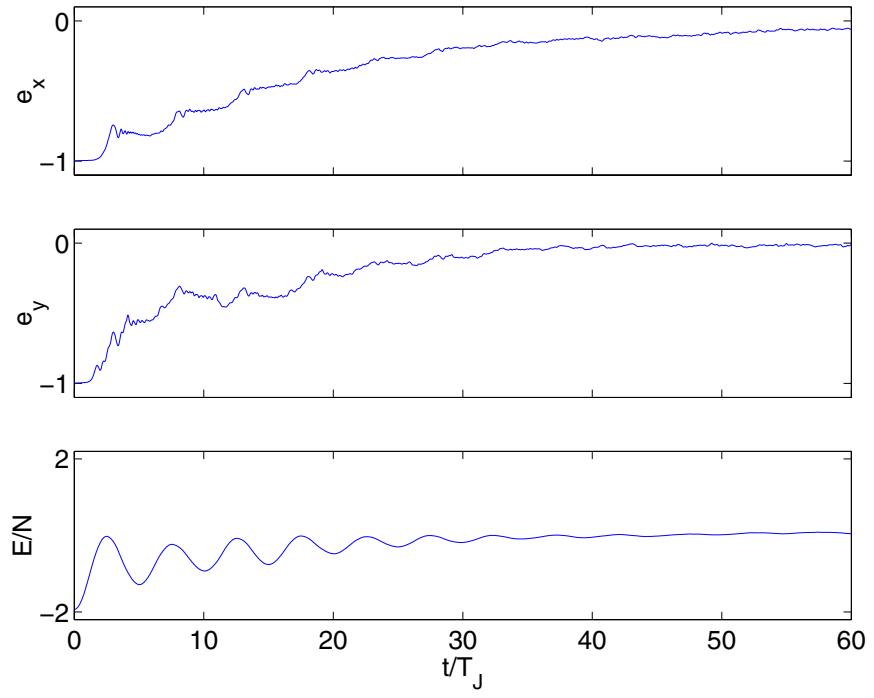

Fig. 3. Bloch dynamics for $d F=0.2 J$ and $\mathbf{F} / F=(0,1)$. The other parameters are the same as in Figure 1.

enter the instability region of the Brillouin zone. However, if the static force is strong enough, the system passes the instability region so quickly that it 'has no time' to decay. (In fact this is a physical argument behind Eq. (8).) In the considered example the strong static force ensures the fast driving along $y$ direction but simultaneously it slowly brings the system to $\kappa_{x}=\pi / 2 d$ along $x$ direction. As soon as this border of instability is reached $\left(t^{*} / T_{J}=J / 4 d F_{x}\right)$, we observe an irreversible decay of the order parameters.

\section{Conclusion}

In summary, we have studied $\mathrm{BO}$ of a $\mathrm{BEC}$ of atoms in a square lattice for both aligned and misaligned static forces. It is shown that in the case of aligned force the system may be reduced to a one-dimensional chain of mini BECs, which we treat by using the mean-field approach. Then the stability diagram of this effective $1 \mathrm{D}$ system defines the critical magnitude of the static force above which BO are stable, with no excitations of the transverse degrees of freedom. On the contrary, in the unstable regime, $F<F_{c r}$, $\mathrm{BO}$ induced by the static force excite the transverse modes and, as a consequence, one observes BEC destruction and decay of BO. Our studies also illuminate importance of the alignment. The strong $\left(F>F_{c r}\right)$ but slightly misaligned force is shown to slowly intrigue the transverse modes, which destabilize $\mathrm{BO}$ after some well-defined transient time. However, if misalignment is large, BO appear to be stable again. This case corresponds to the quantum (not mean-field) regime of BO, where they are modulated with the frequency defined by the microscopic interaction constant.

Fruitful discussions with A. Buchleitner and financial support by Deutsche Forshungsgemeinschaft under SPP1116 are gratefully acknowledged. 


\section{References}

1. B.P. Anderson, M.A. Kasevich, Science 282, 1686 (1998)

2. M. Jone-Lasinio et al., Phys. Rev. Lett. 91, 230406 (2003); C. Sias et al., Phys. Rev. Lett. 98, 120403 (2007)

3. M. Raizen, C. Salomon, Q. Niu, Phys. Today 50, 30 (1997)

4. O. Morsch et al., Phys. Rev. Lett. 87, 140402 (2001)

5. M. Gustavsson et al., e-print arXiv:0710.5083

6. M. Fattori et al., e-print arXiv:0710.5131

7. M. Glück, A.R. Kolovsky, H.J. Korsch, Phys. Rep. 366, $103(2002)$

8. O. Morsch, M. Oberthaler, Rev. Mod. Phys. 78, 179 (2006)

9. V.A. Brazhnyi, V.V. Konotop, Mod. Phys. Lett. B 18, 627 (2004)

10. A.R. Kolovsky, H.J. Korsch, Int. J. Mod. Phys. 18, 1235 (2004)

11. A.R. Kolovsky, H.J. Korsch, Phys. Rev. A 67, 063601 (2003)
12. D. Witthaut, F. Keck, H.-J. Korsch, S. Mossmann, New J. Phys. 6, 41 (2004)

13. M. Snoek, W. Hofstetter, Phys. Rev. A 76, 051603 (2007)

14. H. Trompeter et al., Phys. Rev. Lett. 96, 053903 (2006)

15. A. Widera, I. Bloch, private communication

16. A.R. Kolovsky, e-print arXiv: cond-mat/0412195

17. The condition $\Delta>d F$ modifies the displayed inequality as $\Delta>d F \gg J>W$. For a given (for example, gravitational) force one satisfies the first two inequalities by simply increasing the lattice depth, while the last inequality may require a usage of the Feshbach resonance

18. A. Smerzi et al., Phys. Rev. Lett. 89, 170402 (2002)

19. For a different problem this period was observed in the experiment by M. Greiner, O. Mandel, T.W. Hänsch, I. Bloch, Nature 419, 51 (2002)

20. Yi Zheng, M. Kostrun, J. Javanainen, Phys. Rev. Lett. 93, 230401 (2004) 